03

\title{
О фокусировке широкополосных терагерцовых импульсов
}

\author{
() В.Л. Малевич ${ }^{1}$, Г.В. Синицын ${ }^{1}$, Н.Н. Розанов ${ }^{2,3,4}$ \\ ${ }^{1}$ Институт фризики НАН Беларуси, \\ 220072 Минск, Беларусь \\ ${ }^{2}$ Государственный оптический институт им. С.И. Вавилова, \\ 199053 Санкт-Петербург, Россия \\ ${ }^{3}$ Университет ИТМО, \\ 197101 Санкт-Петербург, Россия \\ ${ }^{4}$ ФТИ имени А.Ф. Иоффре, \\ 194021 Санкт-Петербург, Россия \\ e-mail: v.malevich@ifanbel.bas-net.by, nnrosanov@mail.ru
}

Поступила в редакцию 06.06.2019 г.

В окончательной редакции 06.06.2019 г.

Принята к публикации 18.06.2019 г.

Теоретически исследованы особенности фокусировки линзой широкополосного ТГц импульса с гауссовым поперечным распределением амплитуды. В квазиоптическом приближении получены выражения для пространственно-временного распределения поля на оси пучка и в фокальной плоскости линзы. Показано, что для эффективной фокусировки необходимо использовать линзы с фокусным расстоянием, гораздо меньшим характерной длины дифракции на частоте, соответствующей спектральному максимуму ТГц импульса.

Ключевые слова: терагерцовый импульс, фокусировка,гауссов пучок, дифракция.

DOI: 10.21883/OS.2019.10.48374.213-19

\section{Введение}

В последнее время широкое распространение получил метод импульсной когерентной терагерцовой (ТГц) спектроскопии $[1,2]$. Данный метод используется для исследования спектральных особенностей полупроводников, диэлектриков, полимеров и биологических тканей в области частот, находящейся между СВЧ и дальним инфракрасным диапазонами. Спектральные характеристики определяют путем измерения временной зависимости поля сверхкороткого ТГц импульса, отраженного или прошедшего через исследуемый объект. Сравнивая спектр измеренного ТГц импульса со спектром опорного ТГц сигнала можно восстановить частотные зависимости коэффициента поглощения и показателя преломления исследуемого объекта.

В методе импульсной ТГц спектроскопии для зондирования исследуемого объекта используются широкополосные сфокусированные ТГц импульсы пикосекундной длительности, состоящие из 1-2 осцилляций поля. Из-за сравнительно большой длины волны существенное влияние на процессы распространения, преобразования и фокусировки этих импульсов оказывают дифракционные эффекты. В отличие от узкополосных импульсов здесь важную роль играет зависимость дифракционной длины от частоты излучения. Из-за дифракционного расплывания пучка этот эффект приводит к пространственному разделению спектральных компонент широкополосного ТГц импульса в процессе его распространения в свободном пространстве.
Влияние дифракции на распространение оптических сверхкоротких импульсов в свободном пространстве, а также их преобразование оптическими фокусирующими элементами рассматривались в работах [3-6]. Было показано, что дифракционные эффекты, обусловленные неравенством дифракционных длин спектральных компонент, возрастают с уменьшением длительности импульса и становятся особенно заметными для импульсов, длительность которых сравнима с периодом оптических колебаний.

В работах [7-10] исследовались особенности распространения и фокусировки широкополосных ТГц импульсов с гауссовым поперечным распределением амплитуды. Было отмечено, что для импульсов, состоящих из нескольких осцилляций поля, зависимость дифракционной длины от частоты приводит к значительному искажению его пространственно-временной формы.

В настоящей работе в квазиоптическом скалярном приближении получены выражения для электрического поля прошедшего через линзу широкополосного ТГц импульса с аксиальносимметричным поперечным распределением амплитуды. Рассчитано изменение пространственно-временного распределения поля ТГц биполярного импульса с гауссовым поперечным профилем при его фокусировке.

\section{Теоретическая модель}

Для простоты предположим, что на входе линзы в плоскости $(z=0)$ волновой фронт падающего вдоль 
оси $z$ ТГц импульса является плоским. Действие тонкой линзы сводится к неоднородной по сечению пучка фазовой задержке, которая приводит к искривлению волнового фронта прошедшего импульса. Пространственновременное распределение поля импульса на выходе линзы можно записать в виде

$$
E(t, r, z=0)=\int_{-\infty}^{\infty} d \omega E_{0}(\omega) \exp (i \omega t) U(r) \exp \left(\frac{i \omega r^{2}}{2 c f}\right)
$$

где $f$ - фокусное расстояние линзы, $c$ - скорость света в вакууме, $r$ - радиальная координата в цилиндрической системе координат с осью $z$ вдоль направления распространения импульса. Функция $U(r)$ описывает аксиально-симметричное распределение поля импульса в поперечном сечении, $E_{0}(\omega)=(1 / 2 \pi) \int d t E_{0}(t) \exp (-i \omega t)$ - фурье-спектр начальной временной формы ТГц импульса $E_{0}(t)$. В квазиоптическом скалярном приближении электрическое поле импульса за линзой представляется как

$$
\begin{aligned}
E(t, r, z)= & \frac{i}{2 \pi z c} \int_{-\infty}^{\infty} d \omega \int_{-\infty}^{\infty} d^{2} r^{\prime} \omega E_{0}(\omega) \exp [i \omega(t-z / c)] \\
& \times U\left(r^{\prime}\right) \exp \left(\frac{i \omega r^{\prime 2}}{2 c f}-\frac{i \omega\left(\mathbf{r}-\mathbf{r}^{\prime}\right)^{2}}{2 c z}\right)
\end{aligned}
$$

Интегрирование по частоте позволяет привести выражение (2) к виду

$$
\begin{aligned}
E(t, r, z)= & \frac{1}{2 \pi z c} \frac{d}{d t} \int_{-\infty}^{\infty} d^{2} r^{\prime} U\left(r^{\prime}\right) \\
& \times E_{0}\left[t-\frac{z}{c}+\frac{1}{2 c}\left(\frac{r^{\prime 2}}{f}-\frac{\left(\mathbf{r}-\mathbf{r}^{\prime}\right)^{2}}{z}\right)\right] .
\end{aligned}
$$

На оси пучка $(r=0)$ из выражения (3) получается известный результат $[8,9]$

$$
E(t, z=0, z=f)=\frac{1}{2 \pi f c} \frac{d E_{0}(t-f / c)}{d t} \int_{-\infty}^{\infty} d^{2} f^{\prime} U\left(r^{\prime}\right),
$$

т. е. поле в фокусе линзы определяется производной по времени от начальной временной формы ТГц импульса и для аксиально-симметричных пучков не зависит от его поперечной пространственной структуры. Второе слагаемое в квадратных скобках в выражении (3) описывает добавку к времени запаздывания импульса, возникающую из-за кривизны волнового фронта. На оси пучка это время составляет величину порядка $\left(w^{2} / 2 c\right)(1 / z-1 / f)$ $(w$ - характерная поперечная ширина пучка). В зависимости от положения точки наблюдения относительно фокуса полное время запаздывания огибающей импульса может быть как больше, так и меныше $z / c$.
Далее ограничимся случаем гауссова пучка. После подстановки $U(r)=\exp \left(-r^{2} / w^{2}\right)$ в (2) и интегрирования по сечению пучка для ТГц поля получаем следующее выражение:

$$
\begin{aligned}
E(t, r, z)= & \int_{-\infty}^{\infty} d \omega \frac{E_{0}(\omega) \exp \left[i \omega\left(t-z / c-r^{2} / 2 x c\right)\right]}{\left(1-z / f-i 2 z c / \omega w^{2}\right)} \\
& \times \exp \left[i \frac{r^{2} \omega}{2 z c\left(1-z / f-i 2 z c / \omega w^{2}\right)}\right]
\end{aligned}
$$

Распределение поля в фокальной плоскости линзы можно получить из (5) в виде интеграла свертки по времени:

$$
\begin{aligned}
E(t, r, z & =f)=\frac{w}{2 r \sqrt{\pi}} \frac{d}{d t} \int_{-\infty}^{\infty} d t^{\prime} \\
& \times E_{0}\left(t-t^{\prime}-\frac{f}{c}-\frac{r^{2}}{2 c f}\right) \exp \left[-\frac{f^{2} c^{2} t^{\prime 2}}{r^{2} w^{2}}\right] .
\end{aligned}
$$

Из выражений (5), (6) видно, что из-за различия дифракционных длин спектральных компонент происходит „перепутывание“ пространственных и временных зависимостей импульса. В пределе $r \rightarrow 0$, т. е. на оси пучка, из (6) следует выражение

$$
E(t, r=0, z=f)=\frac{w^{2}}{2 c f} \frac{d E_{0}(t-f / c)}{d t},
$$

согласующееся с (4).

Отметим, что использование квазиоптического приближения накладывает ограничение на ширину ТГц пучка $w \gg c / \omega$ и поэтому в области низких частот $(\omega<c / w)$ данное приближение, строго говоря, неприменимо. Однако для типичного ТГц импульса, генерируемого фемтосекундным оптическим излучением, вес спектральных компонент с частотами $<100 \mathrm{GHz}$ мал и поэтому для пучков с шириной $w>1 \mathrm{~mm}$ использование квазиоптического приближения оправдано.

\section{Численные расчеты и обсуждение результатов}

Пространственно-временное распределение поля фокусируемого широкополосного ТГц импульса рассчитывалось численно из выражения (5). В расчетах использовалась временная зависимость поля ТГц импульса в виде

$$
E_{0}(t)=\left(\gamma e^{-\gamma t}-\delta e^{\delta t}\right) /\left(e^{-\gamma t}+e^{\delta t}\right)^{2} .
$$

Данное выражение достаточно хорошо аппроксимирует типичный профиль биполярных ТГц импульсов, генерируемых фотопроводящей полупроводниковой антенной, возбуждаемой фемтосекундным оптическим излучением. 
Параметры $\gamma$ и $\delta$ определяют ширины положительного и отрицательного пиков ТГц импульса соответственно. В численных расчетах использовались следующие значения параметров: $w=0.5 \mathrm{~cm}, \gamma=10 \mathrm{ps}^{-1}$, $\delta=2 \mathrm{ps}^{-1}$.

На рис. 1 показано распределение поля ТГц импульса в фокальной плоскости, рассчитанное для линзы с фокусным расстоянием $f=5 \mathrm{~cm}$. Видно, что в некоторые моменты времени на оси пучка формируется провал [10], т.е. радиальное распределение поля импульса не является гауссовым. Рассчитанные временные профили поля ТГц импульса на оси пучка после прохождения линзы приведены на рис. 2. Видно, что в фокусе амплитуда поля возрастает примерно в 4 раза для короткофокусной линзы и остается практически неизменной для линзы с фокусным расстоянием $f=20 \mathrm{~cm}$. Отсутствие фокусировки во втором случае связано с эффектом дифракции, который, как известно [11], играет существенную роль, когда длина дифракции $\omega w^{2} / 2 c$ меньше, чем фокусное расстояние. Для ТГц импульса с выбранными параметрами длина дифракции в спектральном максимуме $(\sim 0.7 \mathrm{THz})$ составляет около $18 \mathrm{~cm}$, т.е. сравнима с фокусным расстоянием длиннофокусной линзы. В данном случае дифракционная расходимость в значительной мере компенсирует эффект геометрической сходимости при искривлении волнового фронта пучка линзой. Из расчетов также следует, что при фокусировке длиннофокусной линзой положение точки, где амплитуда широкополосного ТГц импульса достигает максимума (плоскость перетяжки), не совпадает с положением геометрического фокуса, а смещена в сторону линзы примерно на $3 \mathrm{~cm} \mathrm{(рис.} \mathrm{2).} \mathrm{Следует}$ отметить, что величина этого смещения примерно в 3 раза меньше смещения плоскости перетяжки для монохроматического излучения на частоте спектрального максимума импульса $[10,11]$. При фокусировке линзой с $f=5 \mathrm{~cm}$ сдвиг плоскости перетяжки составляет менее $1 \mathrm{~mm}$, т.е. роль дифракции в данном случае незначительна. Дополнительное запаздывание, обусловленное

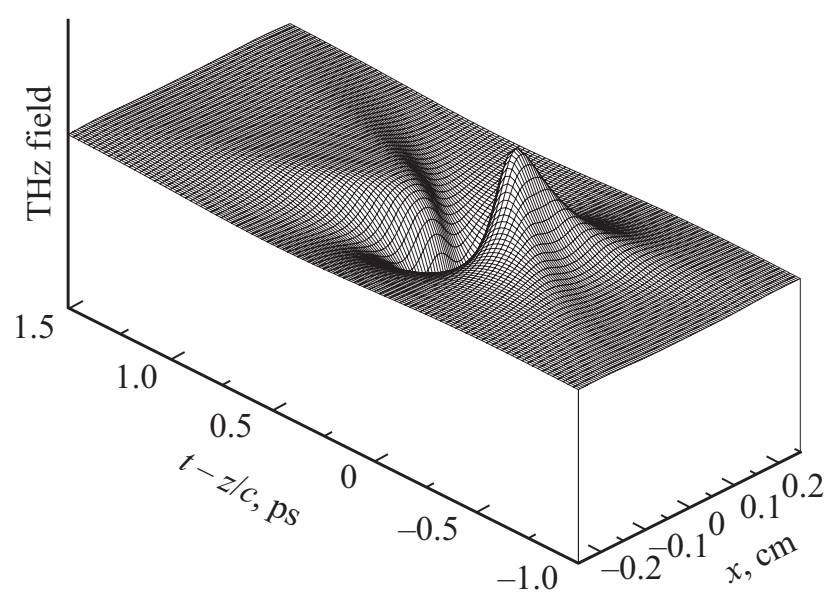

Рис. 1. Радиальное распределение поля ТГц импульса в фокальной плоскости линзы с фокусным расстояние $5 \mathrm{~cm}$.

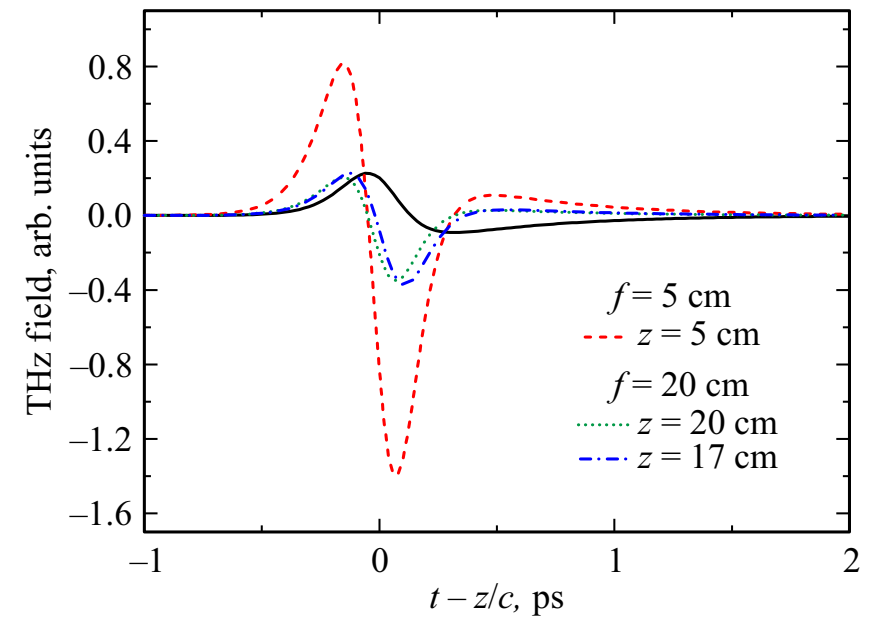

Рис. 2. Временные зависимости электрического поля ТГц импульса на оси пучка. Сплошная линия - начальная форма ТГц импульса перед линзой $(z=0)$.

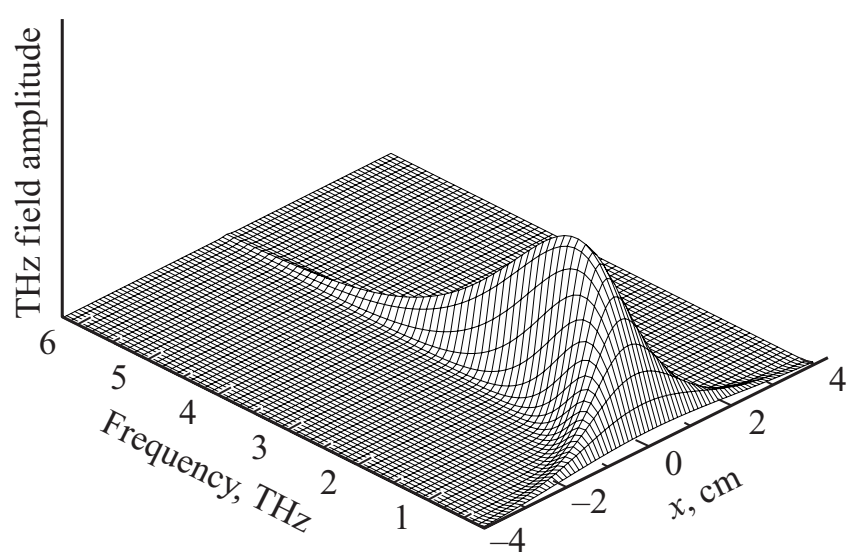

Рис. 3. Радиальное распределение амплитудного спектра ТГц импульса в фокусе линзы с фокусным расстоянием $5 \mathrm{~cm}$.

искривлением волнового фронта, приводит к небольшому сдвигу импульса при смещении точки наблюдения к линзе.

Дифракция препятствует фокусировке и этот эффект проявляется сильнее в области низких частот. Для широкополосного ТГц импульса это означает, что его высокочастотные спектральные компоненты фокусируются сильнее, чем низкочастотные (рис. 3). На оси пучка это приводит к сдвигу максимума амплитудного спектра в сторону более высоких частот. Так, в рассматриваемом нами случае спектральный максимум, расположенный вблизи $0.7 \mathrm{THz}$ для падающего на линзу импульса, сдвигается к частоте $1.3 \mathrm{THz}$. При удалении от оси пучка спектр импульса из-за дифракции обогащается низкочастотными компонентами, и это приводит к смещению спектрального максимума в область более низких частот и, следовательно, увеличению длительности импульса. 


\section{Заключение}

Таким образом, в работе проанализированы особенности фокусировки линзой широкополосного импульса ТГц излучения. Получены аналитические выражения для поля фокусируемого ТГц импульса. Показано, что неравенство дифракционных длин для разных спектральных компонент импульса приводит к искажению пространственно-временного профиля импульса. Расчеты показывают, что для эффективной фокусировки типичного ТГц импульса, генерируемого полупроводниковой фотопроводящей антенной, необходимо использовать линзы с коротким фокусным расстоянием $(<5 \mathrm{~cm})$.

\section{Конфликт интересов}

Авторы заявляют, что у них нет конфликта интересов.

\section{Список литературы}

[1] Yun-Shik Lee. Principles of Terahertz Science and Technology. NY:: Springer, 2009. 340 p.

[2] Си-Чен Чжсан, Джингджю Шю. Терагерцовая фотоника. М.-Ижевск: ИКИ, 2016. 334 с. (Перевод с англ.: X.C. Zhang, J. Xu. Introduction to THz Wave Photonics. NY.: Springer, 2009. 246 p.)

[3] Ахманов С.А., Выслоух В.А., Чиркин А.С. Оптика фемтосекундных лазерных импульсов. М.: Наука, Физматлит, 1988. 312 c.; Akhmanov S.A., Vysloukh V.A., Chirkin A.S. Optics of Femtosecond Laser Pulses, American Institute of Physics, New York, 1992.

[4] Луговой В.Н. // Письма в ЖЭТФ. 1974. Т. 19. № 3. Р. 176; Lugovoi V.N. // JETP Lett. 1974. V. 19. N 3. P. 110.

[5] Christov I.P. // Opt. Commun. 1985. V. 53. N 6. P. 364. doi 10.1016/0030-4018(85)90018-5

[6] Christov I.P. // Opt. Quant. Electron. 1985. V. 17. N 5. P. 353. doi 10.1007/BF00620399

[7] Kaplan A.E. // J. Opt. Soc. Am. B. 1998. V. 15. N 3. P. 951. doi 10.1364/JOSAB.15.000951

[8] Gurtler A., Winnewisser C., Helm H., Jepsen P.U. // J. Opt. Soc. Am. A. 2000. V. 17. N 1. P. 74. doi 10.1364/JOSAA.17.000074

[9] Hattori T., Rungsawang R., Ohta K., Tukamoto K. // Jpn. J. Appl. Phys. 2002. V. 41. N 8. P. 5198.

[10] Розанов Н.Н., Сочилин Г.Б., Федоров С.В., Шацев А.Н., Малевич В.Л., Синицын Г.В. // Опт. и спектр. 2017. Т. 123. № 1. C. 146; Rosanov N.N., Sochilin G.B., Fedorov S.V., Shatsev A.N., Malevich V.L., Sinitsyn G.V. // Opt. Spectrosc. 2017. V. 123. N 1. P. 132.

[11] Виноградова М.Б., Руденко О.В., Сухоруков А.П. Теория волн. М.: Наука, Физматлит. 1979. 384 с. 